\title{
Epidemiology of Hypertension Stages in Two Countries in Sub-Sahara Africa: Factors Associated with Hypertension Stages
}

\author{
Kirubel Zemedkun Gebreselassie ${ }^{1}$ and Mojgan Padyab ${ }^{2}$ \\ ${ }^{1}$ Epidemiology and Global Health, Department of Public Health and Clinical Medicine, Umeå University, 90185 Umeå, Sweden \\ ${ }^{2}$ Centre for Population Studies, Ageing and Living Conditions Programme, Umeå University, Umeå, Sweden \\ Correspondence should be addressed to Kirubel Zemedkun Gebreselassie; kirubelgebresilassie@yahoo.com
}

Received 29 June 2014; Revised 20 October 2014; Accepted 7 November 2014

Academic Editor: Olugbenga Ogedegbe

Copyright ( 2015 K. Z. Gebreselassie and M. Padyab. This is an open access article distributed under the Creative Commons Attribution License, which permits unrestricted use, distribution, and reproduction in any medium, provided the original work is properly cited.

\begin{abstract}
Studies using the revised hypertension classification are needed to better understand epidemiology of hypertension across full distribution. The sociodemographic, biological, and health behavior characteristics associated with different stages of hypertension in Ghana and South Africa (SA) were studied using global ageing and adult health (SAGE), WAVE 1 dataset. Blood pressure was assessed for a total of 7545 respondents, 2980 from SA and 4565 from Ghana. Hypertension was defined using JNC7 blood pressure classification considering previous diagnosis and treatment. Multivariate multinomial logistic regression analysis using Stata version 12 statistical software was done to identify independent predictors. The weighted prevalence of prehypertension and hypertension in Ghana was $30.7 \%$ and $42.4 \%$, respectively, and that of SA was $29.4 \%$ and $46 \%$, respectively, showing high burden. After adjusting for the independent variables, only age ( $\mathrm{OR}=1.32,95 \% \mathrm{CI}: 1.14-1.53)$, income (OR $=1.9,95 \% \mathrm{CI}: 1.04-3.47)$, and $\mathrm{BMI}(\mathrm{OR}=1.16,95 \% \mathrm{CI}$ : 1.1-1.22) remained independent predictors for stage 1 hypertension in Ghana, while, for SA, age $(\mathrm{OR}=$ 2.27, 95\% CI: 1.53-3.36), sex (OR = 0.28, 95\% CI: 0.08-1), and BMI (OR = 1.15, 95\% CI: 1.07-1.25) were found to be independent predictors of stage 1 hypertension. Healthy lifestyle changes and policy measures are needed to promptly address these predictors.
\end{abstract}

\section{Introduction}

Worldwide prevalence estimates for hypertension may be as much as 1 billion individuals, and approximately 7.1 million deaths per year may be attributable to hypertension. The World Health Organization reports that suboptimal systolic blood pressure (SBP) $>115 \mathrm{mmHg}$ is responsible for 62 percent of cerebrovascular disease and 49 percent of ischemic heart disease (IHD), with little variation by sex [1]. Hypertension has been identified as the leading risk factor for developing congestive heart failure [2], stroke [3], chronic kidney disease, and end stage renal disease [4] and is ranked third as a cause of disability-adjusted life-years [5].

The risk of developing these complications depends on the level of elevated blood pressure and has been seen in all age groups starting from blood pressure as low as SBP 115 and DBP of 75 [6]. Data from observational studies involving more than 1 million individuals have also indicated that death from both IHD and stroke increases progressively and linearly from levels as low as $115 \mathrm{mmHg}$ SBP and $75 \mathrm{mmHg}$
DBP upward especially in individuals ranging from 40 to 89 years of age, indicating need for new blood pressure classification [6]. The risk of coronary heart disease increased significantly in the high range prehypertension individuals (SBP 130-139 or DBP $85-89 \mathrm{mmHg}$ ) but not in the low range prehypertensive population (SBP from 120 to 129 or DBP 80 to $84 \mathrm{mmHg}$ ) [7].

Because of the new data on lifetime risk of hypertension and the highly increased risk of cardiovascular morbidity associated with levels of BP previously considered to be normal, the JNC 7 report has introduced a new classification that includes the term "prehypertension" for those with BPs ranging from 120 to $139 \mathrm{mmHg}$ systolic and/or 80 to $89 \mathrm{mmHg}$ diastolic. This new designation is intended to identify those individuals in whom early intervention by adoption of healthy lifestyles could reduce BP, decrease the rate of progression of BP to hypertensive levels with age, or prevent hypertension entirely [8]. Robust population-based data using these recent blood pressure categories are still needed 
to confirm prior estimates and inform policy decision makers in Sub-Saharan Africa.

Increasing urbanization has fueled social and economic changes in Sub-Saharan Africa, which have contributed to a surge in noncommunicable disease (NCD), including hypertension [9]. Epidemiological studies on hypertension in this region have been conducted over the years in an attempt to estimate the burden of hypertension, and these have reported variable rates within and between different population groups. In the first national Demographic and Health Survey, of 12,952 randomly selected South Africans aged 15 years, a high risk of hypertension was associated with less than tertiary education, older age groups, overweight and obese people, excess alcohol use, and a family history of stroke and hypertension [10]. Prehypertension was also more common in those aged 35 years compared with those aged $<35$ years and in overweight and obese people compared with people of normal weight [11]. Hypertension was defined in these studies as individuals with self-reported treated hypertension or with an average of 2 blood pressure measurements of at least 140/90 $\mathrm{mmHg}[9,12,13]$.

Prior studies on hypertension mainly focused on these dichotomous definitions of hypertension and did not examine the sociodemographic characteristics and risk factors for hypertension across full distribution of blood pressure. The current study follows the work of Basu and Millet on social epidemiology of hypertension in low- and middle-income countries from World Health Organization's Study on global AGEing and adult health (SAGE) [13]. Their work further showed additional variation in hypertension prevalence and social determinants of awareness when categorical definitions of hypertension were used compared to dichotomous definitions. Men had significantly lower probability of hypertension awareness than women at stage 1 , but not at stage 2 [14]. This empirical study with an objective of studying social determinants and risk factors of different stages of hypertension tries to address this question. It determines the prevalence and independent predictors of different stages of hypertension in two countries in Sub-Saharan Africa, Ghana and South Africa.

\section{Methods}

2.1. Study Design and Population. We conducted a crosssectional analysis of the Study on global AGEing and adult health (SAGE), WAVE 1 dataset which is a part of longitudinal survey program in WHO's multicountries study unit. The main SAGE surveys compile comparable longitudinal information on the health and well-being of adult populations and the ageing process from nationally representative samples in six middle- and low-income countries. We used the dataset of two Sub-Saharan countries, Ghana and South Africa. The SAGE study included nationally representative sample of persons aged 50 years and sample from younger adults aged 18-49 years as comparison which were also selected from both countries. Multistage cluster sampling was used [15]. The sampling design entailed two-stage probabilistic sample that yielded national and subnational estimates. In the first stage, a total sample of 600 enumeration areas (EA) in South Africa and 300 (EA) in Ghana were drawn from the master sample and used as the primary selection units (PSU). The second stage of the sample design was the selection of households from the EA's which formed the secondary sampling units. This stage of the process involved georeferenced aerial photograph maps of urbanized areas on which the locations of households were plotted. The total sample size of individuals was targeted to be 1000 people in the age group 18-49 years and 5000 people aged 50 years or older in each country.

2.2. Questionnaire. We recorded information about the household members from household and individual questionnaires; living place variable from the household questionnaire and sociodemographic characteristics, work history, blood pressure measurements, and risk factors including preventive health behaviors were taken from the individual questionnaire. Both the household and individual questionnaires were translated into six local South African and three local languages of Ghana. The respondents were interviewed face to face.

\subsection{Blood Pressure Measurements and Classification. Blood} pressure was measured three times while the respondent seated using automated OMRON R6 Wrist Blood Pressure Monitor, HEM-6000-E, Health Care Europe, B.V., Hoofddorp, The Netherlands. Such digital monitors have been shown to have high degree of agreement with mercury sphygmomanometers for systolic blood pressure [16, 17]. Average blood pressure was calculated arithmetically for the 3 measurements of each systolic and diastolic blood pressure. Missing values were excluded from being included in the study. Blood pressure classification was done using JNC 7 algorithm [1]. Prehypertension was defined as systolic blood pressure (SBP) measurement of $120-139 \mathrm{mmHg}$ or diastolic blood pressure (DBP) of $80-89 \mathrm{mmHg}$. Stage 1 hypertension was defined as SBP of $140-159 \mathrm{mmHg}$ or DBP of $90-$ $99 \mathrm{mmHg}$ and stage 2 as SBP of greater than or equal to $160 \mathrm{mmHg}$ or DBP of greater than or equal to $100 \mathrm{mmHg}$. Accordingly normal pressure was defined as SBP of less than $120 \mathrm{mmHg}$ and DBP of less than $80 \mathrm{mmHg}$.

All respondents were initially asked if they have ever been diagnosed with hypertension and if they did, whether or not they have been taking any kind of drugs or other treatment for the last 2 weeks and last 12 months. Normal blood pressure was defined as SBP $<120$ and DBP $<80 \mathrm{mmHg}$ and either no previous history of diagnosis or not taking antihypertensive medication. Those with SBP of 120 or more and DBP of 80 or more who did not have prior diagnosis nor treatment were designed as to have hypertension. They were subsequently stratified into three mutually exclusive different stages of hypertension. Those who reported as either having a diagnosis of hypertension previously or taking antihypertensive medication were excluded from analysis due to overlapping.

2.4. Sociodemographic Characteristics. All participants older than 18 years, of both sexes, were included in the study 
and classified as living in urban or rural area. Current marital status (married or single that included unmarried, widowed, or separated) was asked about using the individual questionnaire. Educational level was assessed by first asking whether respondents have been in any school, and those who answered yes were asked for the highest educational level completed according to the international standard classification of education [18]. Respondents were asked whether they had ever worked for pay, type of work, and employer. Occupation responses were written verbatim by the interviewer, then coded, and mapped to the International Standard Classifications of Occupations (ISCO) scheme [19]. Classification of income quintiles was based on permanent income estimates derived from household assets and characteristics of the dwelling upon which The 2001 WHO World Health Survey/SAGE WAVE 0 relied [15]. Recent alcohol intake was asked for by asking whether the respondents have consumed alcohol in the past 30 days. Response was documented as yes and no. Current smoking (yes or no), with provision for the collection of information on other forms of smoking apart from cigarettes, such as cigars, pipes, snuff, or chewing smokeless tobacco, was asked about. Question was formulated based on guidelines for controlling and monitoring the tobacco epidemic [20]. Work related and sport or leisure time physical activities were separately asked in a typical week. Vigorous or moderate physical activities required hard or moderate physical effort and caused an increase in breathing or heart rate for at least 10 minutes [21]. Fruit and vegetable servings in typical 24 hours were asked about. Inadequate intake was defined based on WHO recommendations and labeled as less than 5 servings ( $80 \mathrm{~g}$ per serving) on a typical day [22].

2.5. Anthropometric Measurements. Height was measured in centimeters after the respondents took off their shoes, put their feet and heels close together, stood straight, and stood forward with their back, head, and heels touching the wall. Next body weight was measured in kilograms. Body composition and fatness were assessed using WHO body mass index (BMI) derived from measured weight in kilograms and normalized by dividing by height in meters squared. It was categorized as underweight $<18.5$, normal weight 18.5-24.9, overweight 25 to 29.9, and obese greater than or equal to 30 . Since abdominal obesity is highly correlated with atherosclerotic cardiovascular morbidity and mortality than BMI indices [23], waist circumference was also used to measure central obesity. The interviewer identified the top of the hip bone and after making sure the tape measure is parallel to the floor all the way around the body measured waist circumference (WC). The National Cholesterol and Education Program: Adult Treatment Panel III (NCEP: ATP III) guideline was used to designate central obesity: accordingly men with WC measurements greater than or equal to $102 \mathrm{~cm}$ and women with greater than or equal to $88 \mathrm{~cm}$ were considered to have one [24].

2.6. Statistical Analysis. Stata version 12 statistical software was used to analyze data after being cleaned. The individual and household data were merged together. Living place variable was taken from the household data while all the others were included from the individual dataset. To make sure the results of the individual country dataset represent the respective country population, weighting at the country level was done which was available in the SAGE dataset. Individual weights were poststratified according to the 2009 projected population estimates in Ghana and to the 2009 medium midyear population estimates in South Africa [25]. Weighted estimates of different stages of hypertension prevalence were reported as proportions of the actual sample size. Invalid blood pressure measurements such as values of diastolic blood pressure greater than systolic and those in the outliers were considered missing and excluded from analysis.

The associations between sociodemographic, biological, and health behavior variables and stages of hypertension (prehypertension, stage 1, and stage 2) were assessed in a twostep procedure where individuals with the different stages of hypertension were compared separately with those having normal blood pressure. In the first step, each variable was evaluated independently in a bivariate multinomial logistic regression analysis with different stages of hypertension as dependent variable to generate unadjusted OR with respondents' characteristics in each country separately. Those variables with $P$ values less than 0.2 were retained and entered into multinomial logistic regression model in ascending stepwise manner to determine variables that were independently associated with the stages of hypertension. A probability level of $P<0.05$ was considered significant. Age and BMI variables were entered in the multivariable multinomial models as continuous due to fewer numbers of people in the most upper and lower categories. All other variables were retained as categorical.

2.7. Ethical Approval. Informed consent was obtained from each respondent for interviews and measurements of anthropometrics. SAGE study received ethical clearance from $\mathrm{WHO}$ ethical review committee [26].

\section{Results}

The total sample size of the study was 8939, 3974 from South Africa and 4965 from Ghana. Participants who have been diagnosed previously with high blood pressure or who were already taking treatment were excluded from analysis due to overlap and difficulty to stratify them into mutually exclusive hypertension stages. Those with previous diagnosis of hypertension were 587 (12\%) in Ghana and 1,111 (28\%) in South Africa. Individuals taking antihypertensive medications were 396 (8\%) in Ghana and 981 (25\%) in South Africa. Completed interview response in South Africa was 2853 (96\%) and 5057 (99\%) in Ghana. Of these blood pressure was assessed for a total of 7545, 2980 (75\%) respondents from South Africa and 4565 (92\%) from Ghana which was included for analysis (Table 1). A higher number of study participants in Ghana were males (50.2\%) compared to $48.5 \%$ in South Africa. More people lived in Urban areas in South Africa (69.7\%) compared to only $44.4 \%$ in Ghana. In South Africa, 
TABLE 1: Sociodemographic, health behavior, and biological characteristics (\%) of study subjects, by country and hypertension stages.

\begin{tabular}{|c|c|c|c|c|c|c|c|c|c|c|}
\hline \multirow{2}{*}{$N=7545$} & \multicolumn{5}{|c|}{ Ghana $(n=4565)$} & \multicolumn{5}{|c|}{ South Africa $(n=2980)$} \\
\hline & Normal & $\begin{array}{l}\text { Pre- } \\
\text { HTN }\end{array}$ & $\begin{array}{c}\text { Stage } 1 \\
\text { HTN }\end{array}$ & $\begin{array}{c}\text { Stage } 2 \\
\text { HTN }\end{array}$ & Total (\%) & Normal & $\begin{array}{l}\text { Pre- } \\
\text { HTN }\end{array}$ & $\begin{array}{c}\text { Stage } 1 \\
\text { HTN }\end{array}$ & $\begin{array}{c}\text { Stage } 2 \\
\text { HTN }\end{array}$ & Total (\%) \\
\hline \multicolumn{11}{|c|}{ Sociodemographic variables } \\
\hline Age groups (years) & $* *$ & & & & & $* *$ & & & & \\
\hline $18-49$ & 28.1 & 32.6 & 20.5 & 18.8 & $762(76.9)$ & 28.2 & 31.7 & 16.2 & 23.9 & $334(79.7)$ \\
\hline $50-59$ & 20.1 & 23.2 & 28.3 & 28.4 & $1532(9.4)$ & 9.1 & 20.9 & 24.9 & 45.1 & $1242(11.1)$ \\
\hline $60-69$ & 18.5 & 24.7 & 23.1 & 33.7 & $1055(6.3)$ & 6.9 & 20.6 & 28.5 & 44.1 & $803(5.7)$ \\
\hline $70-74$ & 18.4 & 26.7 & 23.9 & 30.9 & $837(5.1)$ & 9.5 & 15 & 33.1 & 42.4 & $424(2.5)$ \\
\hline $80+$ & 19.0 & 27.6 & 24.5 & 29 & $374(2.3)$ & 5.8 & 20.4 & 29.6 & 44.2 & $171(1.1)$ \\
\hline Sex & & & & & & $*$ & & & & \\
\hline Male & 26.2 & 29.3 & 20.7 & 23.8 & $2466(50.2)$ & 16.4 & 33.9 & 18.5 & 31.2 & $1361(48.5)$ \\
\hline Female & 25.9 & 32.1 & 22.6 & 19.4 & $2099(49.8)$ & 31.3 & 25.1 & 18.4 & 25.3 & $1617(51.5)$ \\
\hline Living place & & & & & & $*$ & & & & \\
\hline Urban & 23.6 & 30.3 & 24 & 22.2 & $1747(44.4)$ & 28.3 & 28.6 & 18.3 & 24.9 & $1890(69.7)$ \\
\hline Rural & 28 & 31.1 & 19.8 & 21.1 & $2818(55.6)$ & 14.7 & 31.4 & 18.2 & 35.7 & $1084(30.3)$ \\
\hline \multicolumn{11}{|l|}{ Marital status } \\
\hline Married & 25.6 & 28.1 & 23.8 & 22.5 & $1758(26.6)$ & 28.9 & 29.1 & 19.5 & 22.6 & $1346(46.5)$ \\
\hline Single & 26.3 & 31.8 & 20.8 & 21.1 & $2782(73.4)$ & 20 & 29.4 & 17.8 & 32.8 & $1578(53.6)$ \\
\hline Educational level & $*$ & & & & & $* *$ & & & & \\
\hline No school & 21.9 & 27.6 & 22.1 & 28.4 & $2337(31.1)$ & 7.7 & 20.8 & 22.4 & 49.1 & $647(7.3)$ \\
\hline$<6 \mathrm{yrs}$ & 29.3 & 30.3 & 23.5 & 17 & $498(12.8)$ & 5.5 & 23.4 & 22.2 & 48.9 & $556(13.9)$ \\
\hline Primary & 26.4 & 29.3 & 24.8 & 19.6 & $567(20.1)$ & 10.4 & 28.9 & 22 & 38.7 & $557(13.9)$ \\
\hline Secondary & 21.8 & 41.3 & 21 & 15.8 & $244(10.9)$ & 35.2 & 26.2 & 17.4 & 21.3 & $391(27.7)$ \\
\hline High school & 29.5 & 32.3 & 18.2 & 20.1 & $737(20.7)$ & 40.3 & 19.2 & 18.4 & 22.1 & $221(29.1)$ \\
\hline University & 36.8 & 28.8 & 18.2 & 16.3 & $154(4.4)$ & 41.7 & 27.1 & 11.6 & 19.6 & $143(8.1)$ \\
\hline Occupation & $*$ & & & & & & & & & \\
\hline Public sector & 23.6 & 34.1 & 21.8 & 20.6 & $380(7.2)$ & 26.9 & 30.1 & 10.7 & 32.4 & $395(19.8)$ \\
\hline Private & 26.7 & 20.1 & 24.1 & 29.1 & $180(5.2)$ & 13.4 & 32.7 & 23 & 31 & $1421(53.1$ \\
\hline Self-employed & 25 & 32.3 & 22 & 20.7 & $3564(80.8)$ & 44 & 31.8 & 10.5 & 13.8 & $106(4)$ \\
\hline Informal & 25.2 & 24.0 & 15 & 35.8 & $332(6.8)$ & 33.9 & 16.9 & 18.9 & 30.3 & $592(23.1)$ \\
\hline Income & $* *$ & & & & & & & & & \\
\hline Quintile 1 (poorest) & 33.4 & 28.3 & 21.5 & 16.9 & $939(15.6)$ & 20 & 30 & 21.2 & 28.8 & $640(19.6)$ \\
\hline Quintile 2 & 33 & 29 & 16.9 & 21.1 & $932(18)$ & 28.3 & 23.6 & 22.8 & 25.3 & $631(20)$ \\
\hline Quintile 3 & 19.3 & 31 & 20.8 & 28.8 & $922(19.4)$ & 27.4 & 19.6 & 17.3 & 35.6 & $545(20.2)$ \\
\hline Quintile 4 & 18.8 & 34.8 & 24.6 & 21.8 & $921(22.2)$ & 21.1 & 35.6 & 13 & 30.4 & $551(19.5)$ \\
\hline Quintile 5 (richest) & 28.2 & 29.6 & 23.2 & 19.1 & $845(24.9)$ & 23.7 & 37.7 & 17.7 & 20.9 & $595(20.8)$ \\
\hline \multicolumn{11}{|l|}{ Biological variables } \\
\hline $\operatorname{BMI}\left(\mathrm{kg} / \mathrm{m}^{2}\right)$ & $* *$ & & & & & $* *$ & & & & \\
\hline Underweight & 31 & 33 & 14.4 & 21.5 & $670(9.3)$ & 21.1 & 35.7 & 8.2 & 35 & $130(3.5)$ \\
\hline Normal & 30.6 & 29.4 & 19.8 & 20.2 & $2543(54.4)$ & 35.9 & 35.6 & 12.2 & 16.4 & $777(36.1)$ \\
\hline Overweight & 21.4 & 32.8 & 22.2 & 23.6 & $889(24.2)$ & 32.4 & 21.2 & 21.4 & 25 & $849(30)$ \\
\hline Obese & 10.8 & 30.4 & 33.4 & 25.3 & $444(12.1)$ & 5.7 & 23.2 & 25.7 & 45.4 & $1148(30.5)$ \\
\hline Waist circumference & $*$ & & & & & & & & & \\
\hline Normal & 27.9 & 29.9 & 20.2 & 22 & $3608(76.7)$ & 24.5 & 32 & 18.4 & 25.1 & $1574(63.2)$ \\
\hline Abnormal & 20.2 & 32.8 & 26.2 & 20.9 & $930(23.3)$ & 20 & 25.2 & 20.8 & 34 & $1180(36.9)$ \\
\hline \multicolumn{11}{|c|}{ Health related behaviors } \\
\hline Smoking & $*$ & & & & & & & & & \\
\hline No & 25.9 & 31.4 & 22.2 & 20.5 & $3416(84.1)$ & 26.5 & 32.3 & 16.9 & 24.4 & $1819(67.6)$ \\
\hline
\end{tabular}


TABle 1: Continued.

\begin{tabular}{|c|c|c|c|c|c|c|c|c|c|c|}
\hline \multirow[b]{2}{*}{$N=7545$} & \multicolumn{5}{|c|}{ Ghana $(n=4565)$} & \multicolumn{5}{|c|}{ South Africa $(n=2980)$} \\
\hline & Normal & $\begin{array}{l}\text { Pre- } \\
\text { HTN }\end{array}$ & $\begin{array}{l}\text { Stage } 1 \\
\text { HTN }\end{array}$ & $\begin{array}{c}\text { Stage } 2 \\
\text { HTN }\end{array}$ & Total (\%) & Normal & $\begin{array}{l}\text { Pre- } \\
\text { HTN }\end{array}$ & $\begin{array}{l}\text { Stage } 1 \\
\text { HTN }\end{array}$ & $\begin{array}{c}\text { Stage } 2 \\
\text { HTN }\end{array}$ & Total (\%) \\
\hline Yes & 26.2 & 27.6 & 18.8 & 27.5 & $1143(15.9)$ & 19.1 & 23.2 & 22.2 & 35.6 & $1077(32.4)$ \\
\hline \multicolumn{11}{|l|}{ Current alcohol use } \\
\hline No & 25.9 & 31.7 & 20.7 & 21.7 & $1181(44.3)$ & 25.4 & 21.2 & 31.2 & 22.2 & $347(33.5)$ \\
\hline Yes & 28.2 & 27.2 & 22.7 & 22 & $1492(55.7)$ & 14.9 & 27.8 & 18.5 & 38.8 & $489(66.5)$ \\
\hline Work related physical activity & $*$ & & & & & $*$ & & & & \\
\hline High & 25.5 & 33.5 & 19.9 & 21.2 & $1868(44.7)$ & 30.7 & 15.6 & 27 & 26.7 & $306(12.4)$ \\
\hline Moderate & 29 & 28.7 & 22.5 & 19.7 & $1400(27.5)$ & 33.3 & 28.1 & 14.5 & 24.1 & $852(36.3)$ \\
\hline Low & 23.5 & 28.5 & 23.7 & 24.3 & $1289(27.8)$ & 16 & 33.6 & 19.3 & 31.1 & $1734(51.4)$ \\
\hline Fruit intake/day & $*$ & & & & & $* *$ & & & & \\
\hline $0-1$ & 25.7 & 27.9 & 23.9 & 22.5 & $1726(32.3)$ & 17.6 & 24.9 & 21.1 & 36.4 & $1476(46.3)$ \\
\hline $2-4$ & 28 & 31.9 & 19.8 & 20.3 & $2374(57.8)$ & 29.8 & 34.2 & 17.4 & 18.6 & $1305(50.2)$ \\
\hline$>=5$ & 13.7 & 32.7 & 24.7 & 29 & $402(9.9)$ & 37.8 & 9.2 & 7 & 46 & $79(3.5)$ \\
\hline Vegetable intake/day & $*$ & & & & & $* *$ & & & & \\
\hline $0-1$ & 29.9 & 24.1 & 24.5 & 21.5 & $1112(24.9)$ & 14.9 & 24.8 & 24.4 & 35.9 & $1160(35.7)$ \\
\hline $2-4$ & 24.1 & 32.4 & 21.2 & 22.3 & $3269(74.3)$ & 30.8 & 32.7 & 15.3 & 21.2 & $1592(60.6)$ \\
\hline$>=5$ & 21.8 & 36.2 & 29.8 & 12.2 & $57(0.9)$ & 5.4 & 10.1 & 21.7 & 62.9 & $116(3.7)$ \\
\hline Blood pressure number & 957 & 1205 & 1108 & 1295 & 4565 & 301 & 647 & 738 & 1294 & 2980 \\
\hline Weighted prevalence (\%) & 26.1 & 30.7 & 21.6 & 21.6 & & 24.1 & 29.4 & 18.5 & 28.1 & \\
\hline
\end{tabular}

${ }^{*} P<0.2,{ }^{* *} P<0.05$.

$n=$ number of observations in the sample.

All percentages (\%) are put as weighted estimates of the sample to represent population.

the proportion of obesity was $30.5 \%$ as compared to $12.1 \%$ in Ghana. Income quintile distribution was similar in both countries. Only $26.1 \%$ of the respondents in Ghana and 24.1\% in South Africa fulfilled the definition of normal blood pressure. Prehypertension was more prevalent in Ghana (30.7\%) than South Africa (29.4\%). The weighted prevalence of hypertension (both stages 1 and 2) was $42.4 \%$ in Ghana and $46 \%$ in South Africa. The age group distribution across stages of hypertension was similar in both countries, the majority of prehypertensive individuals being in the age group of 18 to 49 . Obese individuals constituted $45.4 \%$ of stage 2 hypertension in South Africa while they constituted only $25.3 \%$ of stage 2 hypertension in Ghana.

Prehypertension was significantly associated in Ghana with income distribution $(\mathrm{OR}=1.9,95 \% \mathrm{CI}: 1.11-3.23)$ and BMI category $(\mathrm{OR}=2.64,95 \% \mathrm{CI}: 1.11-6.3)$ in the bivariate multinomial analysis when compared with normal blood pressure measurement while in South Africa age $(\mathrm{OR}=2.66$, 95\% CI: $1.34-5.28)$ and educational level (OR $=0.18,95 \%$ CI: 0.05-0.63) only had a significant association (Table 2). Income and BMI remained to have a significant association with stage 1 hypertension. In addition age emerged as a new variable with significant correlation with stage 1 hypertension in Ghana in the bivariate analysis (OR $=1.71,95 \%$ CI: $1.25-$ 2.33). In South Africa stage 1 hypertension was significantly associated with age of the study participants (OR $=6.02,95 \%$ CI: 2.61-13.88), educational level ( $\mathrm{OR}=0.09,95 \% \mathrm{CI}: 0.02-$ 0.49 ), type of occupation ( $\mathrm{OR}=4.32,95 \% \mathrm{CI}$ : $1.06-17.55)$, $\mathrm{BMI}$ category $(\mathrm{OR}=11.72,95 \% \mathrm{CI}: 2.35-58.43)$, and number of vegetable servings per day in the bivariate multinomial analysis (OR $=0.3,95 \% \mathrm{CI}$ : 0.11-0.82). Age, income, and BMI remained to be significantly associated with stage 2 hypertension in the bivariate multinomial analysis in Ghana. In addition educational level (OR $=0.07,95 \% \mathrm{CI}$ : 0.02-0.35) became a significant correlate with stage 2 hypertension. In South Africa factors significantly associated with stage 2 hypertension were age, living place, educational level, and fruit and vegetable intake.

\section{Multinomial Logistic Regression Model with Pooled Multivariable Analysis}

4.1. Ghana. For the multinomial logistic regression analysis, 10 independent variables which were associated with hypertension stages at level of $P$ value $<0.2$ in the bivariate analysis were retained in the model. These were age, educational level, type of occupation, income quintile, smoking status, BMI category, abdominal waist circumference, work related physical activity, and fruit and vegetable intake per day. Those with $P$ values $<0.05$ in the multivariable model were considered statistically significant and were considered independent predictors of hypertension stages (Figure 1). At prehypertension level BMI (OR = 1.08, 95\% CI: 1.03-1.14), income $(\mathrm{OR}=2,24,95 \% \mathrm{CI}: 1.27-3.97)$, and number of vegetable intakes per day were found to be independent predictors after being adjusted for the other variables in the model. Income and BMI remained independent predictors 
TABLE 2: Association between sociodemographic, health behavior, and biological variables with stages of hypertension (pre-HTN, stage 1, and stage 2) compared to normal blood pressure: bivariate multinomial analysis.

\begin{tabular}{|c|c|c|c|c|c|c|}
\hline \multirow{3}{*}{$N=7545$} & \multicolumn{3}{|c|}{ Ghana $(n=4565)$} & \multicolumn{3}{|c|}{ South Africa $(n=2980)$} \\
\hline & Pre-HTN & Stage 1 & Stage 2 & Pre-HTN & Stage 1 & Stage 2 \\
\hline & OR (95\% CI) & OR (95\% CI) & OR $(95 \% \mathrm{CI})$ & OR (95\% CI) & OR $(95 \% \mathrm{CI})$ & OR $(95 \% \mathrm{CI})$ \\
\hline \multicolumn{7}{|l|}{ Age groups (years) } \\
\hline $18-49$ & 1 & 1 & 1 & 1 & 1 & 1 \\
\hline $50-59$ & $1(0.78-1.29)$ & $1.93(1.47-2.54)^{*}$ & $2.12(1.59-2.83)$ & $2.06(1.04-4.09)$ & $4.77(2.13-10.67)$ & $5.88(3.01-11.48)$ \\
\hline $60-69$ & $1.15(0.86-1.54)$ & $1.71(1.25-2.33)^{*}$ & $2.72(2.02-3.67)$ & $2.66(1.34-5.28)$ & $7.19(3.38-15.28)$ & $7.57(3.87-14.82)$ \\
\hline $70-74$ & $1.25(0.94-1.66)$ & $1.78(1.26-2.51)^{*}$ & $2.51(1.8-3.51)$ & $1.4(0.57-3.44)$ & $6.02\left(2.61-13.88^{*}\right)$ & $5.25(2.39-11.5)^{*}$ \\
\hline $80+$ & $1.25(0.8-1.97)$ & $1.77(1.1-2.84)^{*}$ & $2.29(1.47-3.57)^{*}$ & $3.17(1.19-8.43)^{*}$ & $8.95(3.5-22.89)$ & 9.09 (3.77-21.93) \\
\hline \multicolumn{7}{|l|}{ Sex } \\
\hline Male & 1 & 1 & 1 & 1 & 1 & 1 \\
\hline Female & $1.11(0.78-0.58)$ & $1.1(0.75-1.61)$ & $0.82(0.56-1.21)$ & $0.39(0.14-1.09)$ & $0.52(0.19-0.44)$ & $0.43(0.17-1.06)$ \\
\hline \multicolumn{7}{|l|}{ Living place } \\
\hline Urban & 1 & 1 & 1 & 1 & 1 & 1 \\
\hline Rural & $0.86(0.6-1.24)$ & $0.69(0.47-1.03)$ & $0.8(0.51-1.25)$ & $2.12(0.82-5.49)$ & $1.92(0.75-4.88)$ & $2.76(1.14-6.68)^{*}$ \\
\hline \multicolumn{7}{|l|}{ Marital status } \\
\hline Married & 1 & 1 & 1 & 1 & 1 & 1 \\
\hline Single & $1.1(0.71-1.72)$ & $0.85(0.55-1.3)$ & $0.91(0.59-1.4)$ & $1.46(0.49-4.34)$ & $1.32(0.46-3.77)$ & $2.1(0.8-5.52)$ \\
\hline \multicolumn{7}{|l|}{ Educational level } \\
\hline No school & 1 & 1 & 1 & 1 & 1 & 1 \\
\hline$<6 \mathrm{yrs}$ & $0.82(0.45-1.49)$ & $0.8(0.44-1.44)$ & $0.45(0.25-0.8)$ & $1.58(0.38-6.53)$ & $1.39(0.44-4.34)$ & $1.4(0.47-4.15)$ \\
\hline Primary & $0.88(0.52-1.49)$ & $0.93(0.53-1.64)$ & $0.57(0.33-0.98)^{*}$ & $1.03(0.28-3.84)$ & $0.73(0.2-2.66)$ & $0.58(0.17-1.95)$ \\
\hline Secondary & $1.5(0.77-2.9)$ & $0.95(0.48-1.92)$ & $0.56(0.26-1.2)$ & $0.27(0.07-1.05)$ & $0.17(0.05-0.61)$ & $0.09(0.03-0.3)$ \\
\hline High school & $0.87(0.52-1.45)$ & $0.61(0.35-1.07)$ & $0.53(0.3-0.92)$ & $0.18(0.05-0.63)^{*}$ & $0.16(0.04-0.7)$ & $0.09(0.02-0.33)$ \\
\hline University & $0.62(0.25-1.56)$ & $0.49(0.18-1.34)$ & $0.34(0.11-1.06)$ & $0.24(0.05-1.13)$ & $0.09(0.02-0.49)^{*}$ & $0.07(0.02-0.35)^{*}$ \\
\hline \multicolumn{7}{|l|}{ Occupation } \\
\hline Public sector & 1 & 1 & 1 & 1 & 1 & 1 \\
\hline Private & $0.52(0.17-1.57)$ & $0.97(0.29-3.23)$ & $1.25(0.38-4.09)$ & $2.18(0.4-12.03)$ & $4.32(1.06-17.55)^{*}$ & $1.93(0.49-7.64)$ \\
\hline Self-employed & $0.89(0.47-1.72)$ & $0.95(0.49-1.85)$ & $0.95(0.46-1.97)$ & $0.65(0.06-7.18)$ & $0.6(0.07-5.23))$ & $0.26(0.03-2.33)$ \\
\hline Informal & $0.66(0.27-1.62)$ & $0.64(0.27-1.55)$ & $1.63(0.6-4.39)$ & $0.45(0.07-2.99)$ & $1.4(0.22-8.72)$ & $0.74(0.16-3.51)$ \\
\hline \multicolumn{7}{|l|}{ Income } \\
\hline Quintile 1 (poorest) & 1 & 1 & 1 & 1 & 1 & 1 \\
\hline Quintile 2 & $1.04(0.6-1.79)$ & $0.79(0.44-1.41)$ & $1.26(0.74-2.14)$ & $0.56(0.14-2.26)$ & $0.76(0.17-3.43)$ & $0.62(0.18-2.14)$ \\
\hline Quintile 3 & $1.9(1.11-3.23)^{*}$ & $1.67(0.94-2.96)$ & $2.95(1.76-4.95)$ & $0.48(0.1-2.37)$ & $0.6(0.11-3.15)$ & $0.9(0.2-4.12)$ \\
\hline Quintile 4 & $2.19(1.3-3.68)$ & $2.03(1.14-3.64)^{*}$ & $2.29(1.32-3.97)^{*}$ & $1.13(0.28-4.6)$ & $0.58(0.15-2.29)$ & $1(0.27-3.73)$ \\
\hline Quintile 5 (richest) & $1.24(0.72-2.12)$ & $1.27(0.74-2.21)$ & $1.33(0.73-2.42)$ & $1.06(0.25-4.43)$ & $0.7(0.2-2.42)$ & $0.61(0.18-2.06)$ \\
\hline \multicolumn{7}{|l|}{ Biological variables } \\
\hline \multicolumn{7}{|l|}{ BMI $\left(\mathrm{kg} / \mathrm{m}^{2}\right)$} \\
\hline Underweight & 1 & 1 & 1 & 1 & 1 & 1 \\
\hline Normal & $0.9(0.51-1.6)$ & $1.39(0.76-2.55)$ & $0.95(0.53-1.71)$ & $0.59(0.12-2.87)$ & $0.88(0.16-4.68)$ & $0.27(0.05-1.38)$ \\
\hline Overweight & $1.44(0.76-2.74)$ & $2.23(1.09-4.56)^{*}$ & $1.58(0.77-3.26)$ & $0.39(0.07-2.06)$ & $1.71(0.28-10.53)$ & $0.47(0.08-2.77)$ \\
\hline Obese & $2.64(1.11-6.3)^{*}$ & $6.62(2.79-15.69)$ & $3.37(1.37-8.26)^{*}$ & $2.42(0.53-11.03)$ & $11.72(2.35-58.43)^{*}$ & $4.81(0.98-23.54)$ \\
\hline \multicolumn{7}{|l|}{ Waist circumference } \\
\hline Normal & 1 & 1 & 1 & 1 & 1 & 1 \\
\hline Abnormal & $1.52(0.97-2.38)$ & $1.79(1.12-2.87)^{*}$ & $1.31(0.8-2.13)$ & $0.96(0.25-3.75)$ & $1.38(0.38-5.04)$ & $1.66(0.49-5.56)$ \\
\hline \multicolumn{7}{|c|}{ Health related behaviors } \\
\hline \multicolumn{7}{|c|}{ Smoking } \\
\hline No & 1 & 1 & 1 & 1 & 1 & 1 \\
\hline Yes & $0.87(0.56-1.34)$ & $0.83(0.51-1.36)$ & $1.33(0.85-2.07)$ & $1(0.33-3.04)$ & $1.83(0.57-5.84)$ & $2.02(0.69-5.95)$ \\
\hline
\end{tabular}


TABLE 2: Continued.

\begin{tabular}{|c|c|c|c|c|c|c|}
\hline \multirow{3}{*}{$N=7545$} & \multicolumn{3}{|c|}{ Ghana $(n=4565)$} & \multicolumn{3}{|c|}{ South Africa $(n=2980)$} \\
\hline & Pre-HTN & Stage 1 & Stage 2 & Pre-HTN & Stage 1 & Stage 2 \\
\hline & OR $(95 \% \mathrm{CI})$ & OR $(95 \% \mathrm{CI})$ & OR $(95 \% \mathrm{CI})$ & OR $(95 \% \mathrm{CI})$ & OR $(95 \% \mathrm{CI})$ & OR $(95 \% \mathrm{CI})$ \\
\hline \multicolumn{7}{|c|}{ Current alcohol use } \\
\hline No & 1 & 1 & 1 & 1 & 1 & 1 \\
\hline Yes & $1.27(0.77-2.1)$ & $0.99(0.6-1.65)$ & $1.08(0.66-1.76)$ & $0.44(0.1-1.93)$ & $0.98(0.15-6.56)$ & $0.33(0.08-1.42)$ \\
\hline \multicolumn{7}{|c|}{ Work related physical activity } \\
\hline High & 1 & 1 & 1 & 1 & 1 & 1 \\
\hline Moderate & $0.75(0.49-1.15)$ & $1(0.63-1.58)$ & $0.82(0.5-1.33)$ & $1.66(0.35-7.95)$ & $0.5(0.08-2.93)$ & $0.83(0.19-3.62)$ \\
\hline Low & $0.92(0.57-1.5)$ & $1.29(0.75-2.23)$ & $1.24(0.72-2.16)$ & $4.14(0.9-19.13)$ & $1.38(0.26-7.42)$ & $2.24(0.56-8.98)$ \\
\hline \multicolumn{7}{|c|}{ Fruit intake/day } \\
\hline $0-1$ & 1 & 1 & 1 & 1 & 1 & 1 \\
\hline $2-4$ & $1.05(0.72-1.53)$ & $0.76(0.5-1.16)$ & $0.83(0.55-1.25)$ & $0.81(0.28-2.32)$ & $0.49(0.17-1.38)$ & $0.3(0.11-0.81)^{*}$ \\
\hline$>=5$ & $2.2(1.06-4.56)$ & $1.94(0.91-4.14)$ & $2.42(1.29-4.54)$ & $0.17(0.02-1.34)$ & $0.15(0.02-1.03)$ & $0.59(0.07-4.65)$ \\
\hline \multicolumn{7}{|c|}{ Vegetable intake/day } \\
\hline $0-1$ & 1 & 1 & 1 & 1 & 1 & 1 \\
\hline $2-4$ & $1.67(1.09-2.57)$ & $1.07(0.69-1.68)$ & $1.28(0.78-2.12)$ & $0.64(0.24-1.68)$ & $0.3(0.11-0.82)^{*}$ & $0.29(0.11-0.72)^{*}$ \\
\hline$>=5$ & $2.07(0.58-7.43)$ & $1.67(0.36-7.63)$ & $0.78(0.22-2.7)$ & $1.13(0.19-6.74)$ & $2.46(0.29-20.65)$ & $4.86(0.66-35.82)$ \\
\hline
\end{tabular}

Multivariate model AOR 95\% CI for Ghana

Prehypertension Vegetable intake $1.62(1.03-2.54)$

Fruit intake $2.17(0.97-4.85)$ Waist C $0.98(0.55-1.75)$

BMI $1.08(1.03-1.14)$ Smoking 0.96 (0.59-1.56)

Income $2.24(1.27-3.97)$

Occupation 0.43 (0.14-1.32)

Educational level $0.45(0.15-1.35)$ Age 1.09 (0.94-1.25)

Stage 1 hypertension Vegetable intake $1.13(0.67-1.9)$ Fruit intake $0.72(0.44-1.16)$ Waist C $0.76(0.42-1.39)$ BMI $1.16(1.1-1.22)$ Smoking $0.93(0.55-1.57)$ Income $1.9(1.04-3.47)$

Occupation $0.66(0.18-2.4)$ Educational level 0.67 (0.36-1.23) Age $1.32(1.14-1.53)$

Stage 2 hypertension Vegetable intake $1.19(0.71-2)$ Fruit intake 2.96 (1.44-6.08) Waist C $0.66(0.36-1.21)$ BMI $1.14(1.08-1.21)$ Smoking $1.21(0.72-2.01)$ Income 3.52 (2.02-6.12) Occupation $1.02(0.29-3.61)$ Educational level 0.47 (0.24-0.93) Age 1.35 (1.17-1.56)

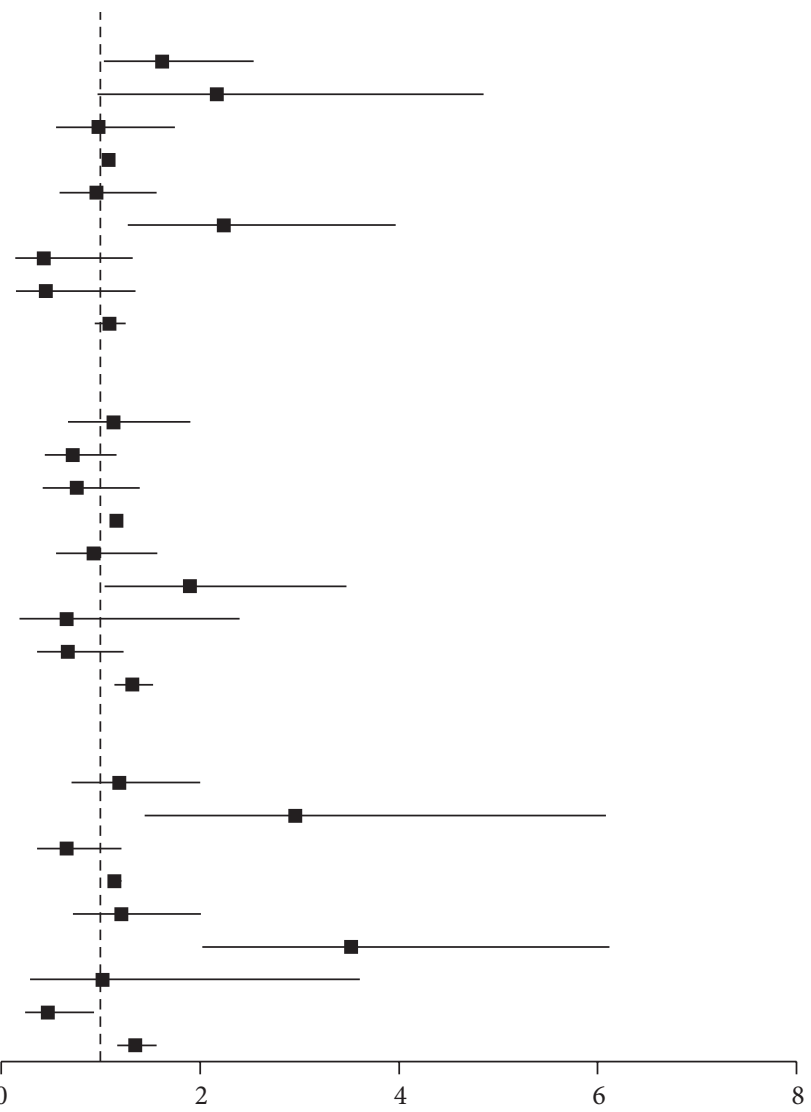

FIgURE 1: Adjusted odds ratios and 95\% confidence intervals for stages of hypertension, Ghana. 


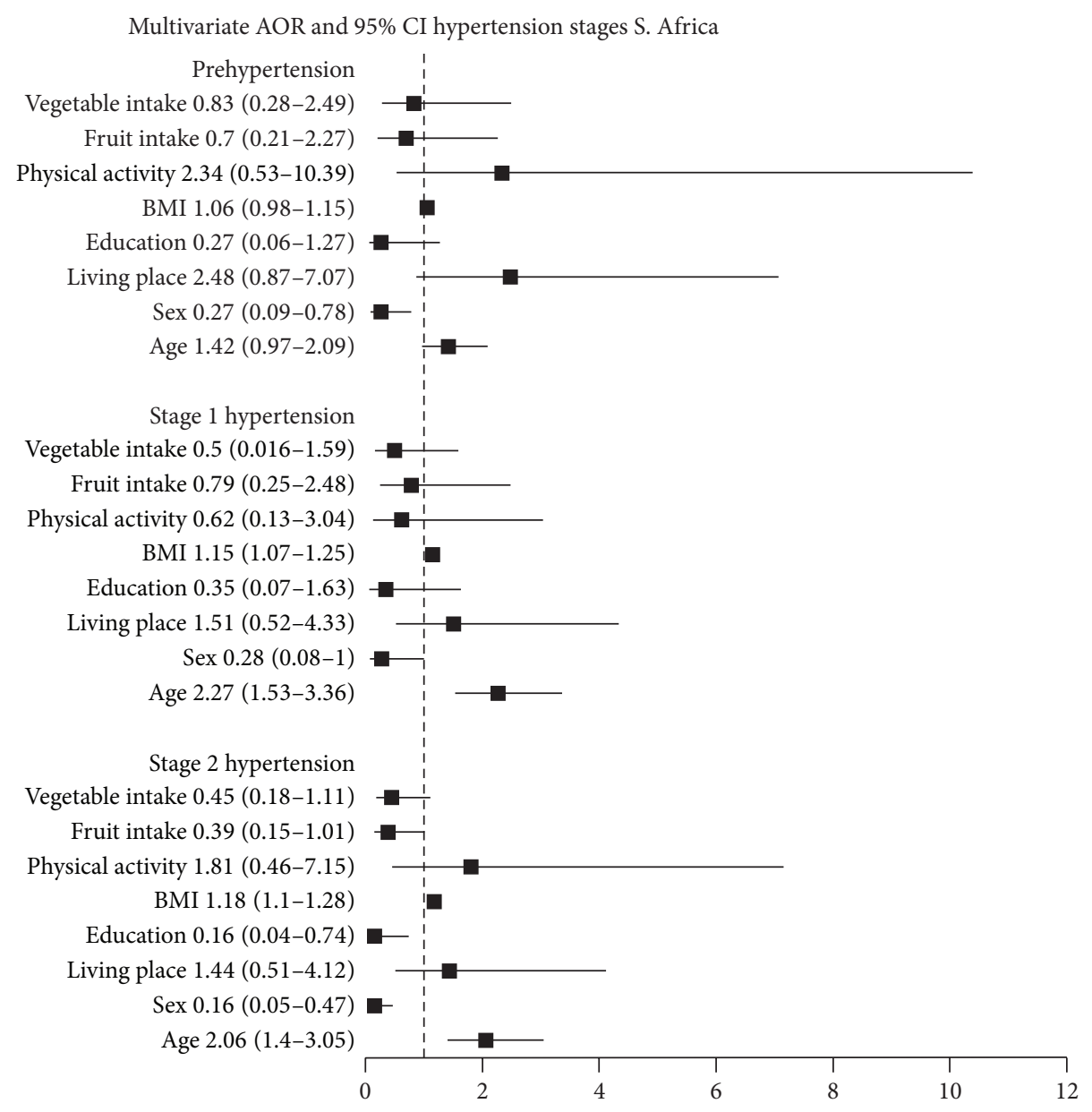

Figure 2: Adjusted odds ratios and 95\% confidence intervals for stages of hypertension, South Africa.

for stage 1 hypertension as well. There was 32\% increased risk of having stage 1 hypertension for every age increase by one year $(\mathrm{OR}=1.32,95 \% \mathrm{CI}: 1.14-1.53)$. Age $(\mathrm{OR}=1.35,95 \%$ CI: $1.17-1.56)$, income (OR $=3.52,95 \% \mathrm{CI}: 2.02-6.12)$, and BMI $(\mathrm{OR}=1.14,95 \% \mathrm{CI}: 1.08-1.21)$ remained as independent predictors for stage 2 hypertension as well. In addition higher educational level emerged as a protective variable against stage 2 hypertension in the model (OR $=0.47,95 \%$ CI: $0.24-$ $0.93)$.

4.2. South Africa. Eight variables which were associated with stages of hypertension in the bivariate analysis at probability level of $P<0.2$ were retained in the multinomial logistic regression model. These were age, sex, living place, educational level, BMI category, work related physical activity, and fruit and vegetable servings per day (Figure 2). Only sex (OR $=0.27,95 \%$ CI: $0.09-0.78$ ) remained independent predictor of prehypertension in the multivariate logistic regression model after being adjusted for the other variables. Age and BMI emerged as independent predictors of stage 1 hypertension $(\mathrm{OR}=2.27,95 \% \mathrm{CI}: 1.53-3.36$ and $\mathrm{OR}=1.15$, 95\% CI: $1.07-$ 1.25). Sex remained as an independent predictor of stage 1 hypertension $(\mathrm{OR}=0.28,95 \% \mathrm{CI}$ : 0.08-1). Being female resulted in $72 \%$ lower risk of having stage 1 hypertension than being male $(\mathrm{OR}=0.28,95 \% \mathrm{CI}$ : 0.08-1). Age (OR $=2.06,95 \%$ CI: $1.4-8.7)$, sex $(\mathrm{OR}=0.16,95 \% \mathrm{CI}: 0.05-0.47)$, and $\mathrm{BMI}$ $(\mathrm{OR}=1.18,95 \% \mathrm{CI}: 1.1-1.28)$ remained independent predictors of stage 2 hypertension in the final model. Educational level emerged as an independent predictor of stage 2 hypertension. Those with high school education tended to have $84 \%$ lower odds of stage 2 hypertension compared to those with no education $(\mathrm{OR}=0.16,95 \% \mathrm{CI}: 0.04-0.74)$.

\section{Discussion}

The study has showed high burden of prehypertension and hypertension stages in the Sub-Saharan African countries, Ghana and South Africa. The weighted prevalence of hypertension (including both stages 1 and 2) was higher in South Africa (46\%) compared to Ghana (42.4\%). The high prevalence of hypertension in the current study is mostly due to higher age distribution of participants. More than $85 \%$ of respondents in Ghana and around 90\% South Africans were in the age group more than 50, as part of the WHO SAGE study. The probability that a middle-aged or elderly individual will develop hypertension in his or her lifetime is 90\% [27], 
explaining the higher prevalence in this study. Higher prevalence of hypertension (77.3\%) than the current study was also reported in hypertension and associated factors in older adults study in South Africa [28]. Comparing the two countries South Africa had higher prevalence of hypertension that could be explained by higher proportion of people living in urban area (69.7\% in South Africa and $44.4 \%$ in Ghana) and increased number of obese people (30.5\% in South Africa and $12.1 \%$ Ghana). In addition lower rate of physical activity and fruit intake per day was reported in South Africa.

The weighted prevalence of prehypertension was higher in Ghana (30.7\%) than South Africa (29.4\%). Higher reports were made from the PURE hypertension trial done on 153,996 adults aged 35 to 70 years, from 628 communities in 3 highincome, 10 upper-middle and low-middle-income, and 4 lowincome countries of $36.8 \%$ [12]. These are groups of people with increased cardiovascular risk but who do not need pharmacologic treatment unless there is another compelling medical indication such as diabetes or chronic kidney disease [29]. They have higher likelihood of progression to overt hypertension and need lifestyle changes as a treatment such as weight reduction, physical activity, and decreased salt intake [30]. Progression from prehypertension to stage 1 hypertension was positively related to male gender, higher waist circumference, and having parents with hypertension in population-based study Keelung, Taiwan [31]. Identifying these individuals has high public health importance as such measures taken could delay or prevent progression or development of hypertension.

The weighted prevalence of stage 1 hypertension in Ghana was higher (21.6\%) than in South Africa (18.5\%) and the weighted estimate of stage 2 hypertension was higher in South Africa (28.1\%) and Ghana (21.6\%). Prior studies on these two countries were mainly done on dichotomized blood pressure classification using SBP of $140 \mathrm{mmHg}$ or DBP of $90 \mathrm{mmHg}$ and more. According to hypertension in South African adults, results from the Demographic and Health Survey, 1998, the prevalence rate of hypertension was $11 \%$ in males and $14 \%$ in females when blood pressure cut-off point $(160 / 95 \mathrm{mmHg})$ was used [32]. In the current study BMI was an independent predictor for prehypertension in both Ghana and South Africa which was a similar finding in the study done in the Ashanti region of Ghana which showed age $(\mathrm{OR}=$ 1.56, 95\% CI: 1.12-2.18; $P<0.01$ ), obesity (OR = 2.71; 95\% CI: $1.40-5.24 ; P<0.001)$, and sex $(\mathrm{OR}=2.36,95 \% \mathrm{CI}: 1.77-3.15$; $P<0.001)$ being independent predictors of prehypertension on multivariable logistic regression [11]. In addition income became one of the strongest predictors for prehypertension in Ghana in our study, with higher income quintiles associated with higher levels of prehypertension. This was similar with household characteristics for older adults and study background from SAGE Ghana WAVE 1, which showed people with the higher income quintiles generally reported more hypertension (income quintile 5 , wealthiest $=26.7 \%$, versus quintile 1 , poorest $=5.5 \%$ ) and received more current and chronic therapy [15]. Other studies in US and Canada have shown the opposite effect; inverse linear relationship between household income and hypertension prevalence in the United States, but no evidence of such a relationship in Canada, was seen due to similar burden of hypertension across different socioeconomic classes [33]. Middle-income was a high correlate in another study in urban India [34]. Getting income data reliably was difficult as income was generated from ho usehold assets and converted later to quintiles, hence requiring cautious interpretation of result. The proportion of both overweight and obese was higher in the 4th and 5th income quintiles than the lower ones (23\% and $30 \%$, resp.) which could explain the high prevalence of prehypertension in the group.

Being in the higher BMI category contributed significantly to having prehypertension and could be related to the low physical activity of the current study participants [35]. Those with the lowest work related physical activity had the highest rates of prehypertension. South Africa has one of the lowest rates of insufficient physical activity (49\% in adult women and $43 \%$ in adult men) compared to global figure of $17 \%$ and Africa's coverage of about $10 \%$ [36]. Some of the explanations were high rate of urbanization, increased mechanized labour, and television watching [37]. The very low report of physical activity contributing to both obesity and prehypertension needs to be addressed in South Africa by clinicians, public health specialists, patients, and policy makers at the government level.

We have tried to see the social determinant and risk factors for different hypertension stages (1 and 2) separately and whether they differ in the two countries and across different hypertension stages in the same country. The study has showed only age, income, and BMI remaining as independent predictors of stage 1 hypertension in Ghana. We found educational level and number of fruit intakes per day having an inverse relationship to stage 1 hypertension in South Africa in the bivariate multinomial analysis. This is similar with the first demographic and health survey study: determinants and treatment of hypertension in South Africa which showed higher risk of having hypertension with less than tertiary education [10]. Adults with no education or less than primary school were more than $50 \%$, and the highest report of stage 1 hypertension was seen in this group in the current study. Curriculum reforms and models to increase opportunity of education in the postapartheid education system are undergoing [38] and should be further strengthened.

Increasing fruit and vegetable intake was seen to have significant blood pressure lowering effect in stage 1 and stage 2 hypertension. This effect was not seen in the final model. The finding is consistent with other trials that demonstrated the greatest benefit of dietary changes at lower stages of hypertension [30]. The DASH (Dietary Approaches to Stop Hypertension) trials introduced the DASH diet which is comprised of four-five servings of fruit, four-five servings of vegetables, two-three servings of low-fat dairy per day, and $<25$ percent fat [39]. Drop in average systolic blood pressure by $11.4 \mathrm{mmHg}$ and diastolic blood pressure by $5.5 \mathrm{mmHg}$ in hypertensive individuals has been seen as early as two weeks, with the DASH diet. Increasing fruit intake is thus highly recommended in individuals especially with lower stages of hypertension to prevent progression of disease. Countries should be working on ways to make availability of fruits accessible and affordable. The weighted estimate of people 
taking the recommended fruit intake was very low (9.9\%) in Ghana and (3.5\%) South Africa. Vegetable intake was even much worse $(0.9 \%)$ in Ghana and $(3.5 \%)$ in South Africa. The wide confidence interval and attenuated beneficial effects of fruits and vegetables could be attributed to the very little proportion of people in these categories making the expected statistical association loose.

Though smoking cigarette was one of the most important risk factors for cardiovascular disorders and acute myocardial infarction in the INTERHEART Africa study [40], it was associated only in the bivariate analysis when studied individually. The effect was lost when multiple variables were retained in the regression model in both countries at every stage of hypertension. The incidence of hypertension increases in those who smoke 15 or more cigarettes per day [41] and could be the reason why strong association was not seen as only less than $2 \%$ individuals (Ghana) and around $7 \%$ (South Africa) smoked more than 15 cigarettes per day. Other studies have also documented "lower blood pressure measurements in those habitual smokers than nonsmokers due to weight loss and some vasodilatory effect of cotinine a metabolite of nicotine" $[42,43]$.

Only increasing age, income, BMI, and educational level were found to be independent risk factors for stage 2 hypertension in the final model in Ghana. There was no significant variation of stage 2 hypertension between sexes in Ghana, although it is known that men had higher systolic blood pressure measurements in early adulthood, while older women have steeper age-related rate of rise [27]. This could be in part due to the higher proportion of women who are overweight and obese in both countries.

This particular study has assessed the burden of hypertension in Sub-Saharan African countries. It focused mainly on risk factors and social determinants across different stages of hypertension among the two countries. This helps to identify newly emerging associated risk factors at different stages of hypertension and helps to be able to recommend measures accordingly. As it is always said "Prevention is better than Cure," we also recommend identifying individuals and treating accordingly at pre- and early hypertension stages, where the maximum benefit of lifestyle changes can be seen. These include having regular and intense physical exercise, reduction of body weight which can decrease average SBP by $5-20 \mathrm{mmHg}$ for every $10 \mathrm{~kg}$ weight loss [1], increasing the opportunity to have basic education for all, and educating people with higher income in Ghana who are at high risk of prehypertension and subsequent overt hypertension due to probable acculturation to change their lifestyle. Government bodies at policy level and health specialists need to design methods to improve diagnosis and treatment of hypertension at earlier stages in respective countries.

\section{Limitation}

One of the major limitations of the current study was high number of missing and some invalid values in the original dataset. Missing and invalid blood pressure measurements accounted for $8 \%$ in Ghana and 25\% in South Africa. They were excluded during the data cleaning period and not included in analysis. Alcohol consumption was an independent variable with the highest missing values (63\%) in Ghana and (35\%) in South Africa. This could be the reason for not seeing the expected protection from moderate dose of alcohol against hypertension and increased risk from excess dose [44]. The other components of therapeutic lifestyle changes that include intake of salt, saturated fat, and amount of calories [45] were not included in the SAGE questionnaire and their association with hypertension was not studied. The current study as any other cross-sectional studies determines only associations that are statistically significant without inferring causality. Further cohort studies that examine risk and causality are recommended. And finally using the JNC 7 blood pressure classification that was originally designed for US population and using its blood pressure cut-off points for Sub-Saharan population could impose risk of overgeneralization. Blood pressure cut-off points at which cardiovascular morbidity starts should be looked for in Sub-Saharan Africa context and guidelines should develop in the future.

\section{Abbreviations}

$\begin{array}{ll}\text { AOR: } & \text { Adjusted odds ratio } \\ \text { BP: } & \text { Blood pressure } \\ \text { BMI: } & \text { Body mass index } \\ \text { CVD: } & \text { Cardiovascular disorder } \\ \text { CI: } & \text { Confidence interval } \\ \text { DASH: } & \text { Dietary approaches to stop } \\ & \text { hypertension } \\ \text { DBP: } & \text { Diastolic blood pressure } \\ \text { EA: } & \text { Enumeration area } \\ \text { HTN: } & \text { Hypertension } \\ \text { IHD: } & \text { Ischemic heart disease } \\ \text { ISCO: } & \text { International standard classification of } \\ & \text { occupation } \\ \text { JNC 7: } & \text { Joint national committee } 7 \text { for } \\ & \text { hypertension } \\ \text { NCD: } & \text { Noncommunicable disease } \\ \text { NCEP: } & \text { ATP III: National Cholesterol Education } \\ & \text { Program: Adult Treatment Panel III } \\ \text { OR: } & \text { Odds ratio } \\ \text { PSU: } & \text { Primary selection unit } \\ \text { SA: } & \text { South Africa } \\ \text { SAGE: } & \text { Study on global AGEing and adult } \\ & \text { health } \\ \text { SBP: } & \text { Systolic blood pressure } \\ \text { WC: } & \text { Waist circumference } \\ \text { WHO: } & \text { World Health Organization. }\end{array}$

\section{Conflict of Interests}

The authors declare that there is no conflict of interests regarding the publication of this paper. 


\section{Acknowledgments}

The authors wish to thank the WHO SAGE study group, Umeå International School of Public Health (UISPH), particularly Kjerstin Dahlblom, Nawi Ng, and Jennifer Stewart Williams, and Swedish Institute (SI) scholarships.

\section{References}

[1] A. V. Chobanian, G. L. Bakris, H. R. Black et al., "The seventh report of the joint national committee on prevention, detection, evaluation, and treatment of high blood pressure: the JNC 7 report," Journal of the American Medical Association, vol. 289, no. 19, pp. 2560-2572, 2003.

[2] F. Palano, F. Paneni, S. Sciarretta, G. Tocci, and M. Volpe, "The progression from hypertension to congestive heart failure," Recenti Progressi in Medicina, vol. 102, no. 12, pp. 461-467, 2011.

[3] J. A. Staessen, R. Fagard, L. Thijs et al., "Randomised doubleblind comparison of placebo and active treatment for older patients with isolated systolic hypertension," The Lancet, vol. 350, no. 9080, pp. 757-764, 1997.

[4] J. Coresh, G. L. Wei, G. McQuillan et al., "Prevalence of high blood pressure and elevated serum creatinine level in the United States: findings from the third national health and nutrition examination survey (1988-1994)," Archives of Internal Medicine, vol. 161, no. 9, pp. 1207-1216, 2001.

[5] M. E. Hendriks, F. W. N. M. Wit, M. T. L. Roos et al., "Hypertension in Sub-Saharan Africa: cross-sectional surveys in four rural and urban communities," PLoS ONE, vol. 7, no. 3, Article ID e32638, 2012.

[6] S. Lewington, R. Clarke, N. Qizilbash, R. Peto, and R. Collins, "Age-specific relevance of usual blood pressure to vascular mortality: a meta-analysis of individual data for one million adults in 61 prospective studies," The Lancet, vol. 360, no. 9349, pp. 1903-1913, 2002.

[7] L. Shen, H. Ma, M.-X. Xiang, and J.-A. Wang, "Meta-analysis of cohort studies of baseline prehypertension and risk of coronary heart disease," American Journal of Cardiology, vol. 112, no. 2, pp. 266-271, 2013.

[8] C. Lenfant, A. V. Chobanian, D. W. Jones, and E. J. Roccella, "Seventh report of the Joint National Committee on the prevention, detection, evaluation, and treatment of high blood pressure (JNC 7): resetting the hypertension sails," Hypertension, vol. 41, no. 6, pp. 1178-1179, 2003.

[9] P. Kotwani, D. Kwarisiima, T. D. Clark et al., "Epidemiology and awareness of hypertension in a rural Ugandan community: a cross-sectional study," BMC Public Health, vol. 13, no. 1, article 1151, 2013.

[10] K. Steyn, D. Bradshaw, R. Norman, and R. Laubscher, "Determinants and treatment of hypertension in South Africans: the first demographic and health survey," South African Medical Journal, vol. 98 , no. 5, pp. 376-380, 2008.

[11] C. Agyemang and E. Owusu-Dabo, "Prehypertension in the Ashanti region of Ghana, West Africa: an opportunity for early prevention of clinical hypertension," Public Health, vol. 122, no. 1, pp. 19-24, 2008.

[12] C. K. Chow, K. K. Teo, S. Rangarajan et al., "Prevalence, awareness, treatment, and control of hypertension in rural and urban communities in high-, middle-, and low-income countries," Journal of the American Medical Association, vol. 310, no. 9, pp. 959-968, 2013.
[13] W. K. Bosu, "Epidemic of hypertension in Ghana: a systematic review," BMC Public Health, vol. 10, article 418, 2010.

[14] B. D. Capistrant and P. Kowal, "Social epidemiology of hypertension stages," Hypertension, vol. 62, no. 6, article e41, 2013.

[15] R. B. Biritwum, G. Mensah, N. Minicuci et al., "Household characteristics for older adults and study background from SAGE Ghana Wave 1," Global Health Action, vol. 6, no. 1, Article ID 20096, 2013.

[16] A. M. B. Menezes, S. C. Dumith, R. B. Noal et al., "Validity of a wrist digital monitor for blood pressure measurement in comparison to a mercury sphygmomanometer," Arquivos Brasileiros de Cardiologia, vol. 94, no. 3, pp. 345-370, 2010.

[17] H. Takahashi, M. Yoshika, and T. Yokoi, "Validation of Omron RS8, RS6, and RS3 home blood pressure monitoring devices, in accordance with the European Society of Hypertension International Protocol revision 2010," Vascular Health and Risk Management, vol. 9, pp. 265-272, 2013.

[18] S. L. Schneider and I. Kogan, "The International Standard Classification of Education 1997: challenges in the application to national data and the implementation in cross-national surveys," in The International Standard Classification of Education (ISCED-97): An Evaluation of Content and Criterion Validity for 15 European Countries, pp. 13-46, MZES, 2008.

[19] L. Wirth, Copyright International Labour Organization 2001 First published 2001 Second impression 2001 Publications of the International Labour Office enjoy copyright under Protocol 2 of the Universal Copyright Convention, Nevertheless, short excerpts from them may be reproduced without authorization, 2001.

[20] World Health Organization, Guidelines for Controlling and Monitoring the Tobacco Epidemic, World Health Organization, 1998.

[21] T. Armstrong and F. Bull, "Development of the World Health Organization Global Physical Activity Questionnaire (GPAQ)," Journal of Public Health, vol. 14, no. 2, pp. 66-70, 2006.

[22] World Health Organization, Global Health Risks: Mortality and Burden of Disease Attributable to Selected Major Risks, World Health Organization, 2009.

[23] J. I. Recio-Rodriguez, M. A. Gomez-Marcos, M. C. PatinoAlonso, C. Agudo-Conde, E. Rodriguez-Sanchez, and L. GarciaOrtiz, "Abdominal obesity vs general obesity for identifying arterial stiffness, subclinical atherosclerosis and wave reflection in healthy, diabetics and hypertensive," BMC Cardiovascular Disorders, vol. 12, article 3, 2012.

[24] A. M. Gotto, "NCEP ATP III guidelines incorporate global risk assessment," The American Journal of Managed Care, supplement, pp. 1-3, 2003.

[25] W. He, M. N. Muenchrath, and P. R. Kowal, Shades of Gray: A Cross-Country Study of Health and Well-Being of the Older Populations in SAGE Countries, 2007-2010, Economics and Statistics Administration, US Census Bureau, 2012.

[26] N. Naidoo, S. Abdullah, A. Bawah et al., Ageing and Adult Health Status in Eight Lower-Income Countries: the INDEPTH WHOSAGE Collaboration, Global Health Action, 2010.

[27] A. S. Fauci, Harrison's Principles of Internal Medicine, McGrawHill Medical, New York, NY, USA, 2008.

[28] K. Peltzer and N. Phaswana-Mafuya, "Hypertension and associated factors in older adults in South Africa," Cardiovascular Journal of Africa, vol. 24, no. 3, pp. 67-71, 2013.

[29] S. Chaturvedi, "The seventh report of the joint national committee on prevention, detection, evaluation, and treatment of high 
blood pressure (JNC 7): is it really practical?” National Medical Journal of India, vol. 17, no. 4, p. 227, 2004.

[30] L. J. Appel, M. W. Brands, S. R. Daniels, N. Karanja, P. J. Elmer, and F. M. Sacks, "Dietary approaches to prevent and treat hypertension: a scientific statement from the American Heart Association," Hypertension, vol. 47, no. 2, pp. 296-308, 2006.

[31] Y.-H. Chiu, S.-C. Wu, C.-D. Tseng, M.-F. Yen, and T. H.-H. Chen, "Progression of pre-hypertension, stage 1 and 2 hypertension (JNC 7): a population-based study in Keelung, Taiwan (Keelung Community-based Integrated Screening No. 9)," Journal of Hypertension, vol. 24, no. 5, pp. 821-828, 2006.

[32] K. Steyn, T. A. Gaziano, D. Bradshaw, R. Laubscher, and J. Fourie, "Hypertension in South African adults: results from the demographic and health survey, 1998," Journal of Hypertension, vol. 19, no. 10, pp. 1717-1725, 2001.

[33] M. S. Kaplan, N. Huguet, D. H. Feeny, and B. H. McFarland, "Self-reported hypertension prevalence and income among older adults in Canada and the United States," Social Science and Medicine, vol. 70, no. 6, pp. 844-849, 2010.

[34] S. Yadav, R. Boddula, G. Genitta et al., "Prevalence \& risk factors of pre-hypertension \& hypertension in an affluent North Indian population," Indian Journal of Medical Research, vol. 128, no. 6, pp. 712-720, 2008.

[35] S. Mora, I.-M. Lee, J. E. Buring, and P. M. Ridker, "Association of physical activity and body mass index with novel and traditional cardiovascular biomarkers in women," The Journal of the American Medical Association, vol. 295, no. 12, pp. 1412-1419, 2006.

[36] J. Joubert, R. Norman, E. V. Lambert et al., "Estimating the burden of disease attributable to physical inactivity in South Africa in 2000," South African Medical Journal, vol. 97, no. 8, pp. 725-731, 2007.

[37] E. V. Lambert, M. I. Lambert, K. Hudson et al., "Role of physical activity for health in communities undergoing epidemiological transition," World Review of Nutrition and Dietetics, vol. 90, pp. 110-126, 2001.

[38] J. D. Jansen, "Curriculum Reform in South Africa: a critical analysis of outcomes-based education," Cambridge Journal of Education, vol. 28, no. 3, pp. 321-331, 1998.

[39] L. J. Appel, T. J. Moore, E. Obarzanek et al., "A clinical trial of the effects of dietary patterns on blood pressure," Journal of Materials Science: Materials in Medicine, vol. 8, no. 4, pp. 1117$1124,1997$.

[40] K. Steyn, K. Sliwa, S. Hawken et al., "Risk factors associated with myocardial infarction in Africa: the INTERHEART Africa Study," Circulation, vol. 112, no. 23, pp. 3554-3561, 2005.

[41] T. S. Bowman, J. M. Gaziano, J. E. Buring, and H. D. Sesso, "A prospective study of cigarette smoking and risk of incident hypertension in women," Journal of the American College of Cardiology, vol. 50, no. 21, pp. 2085-2092, 2007.

[42] K. A. Perkins, L. H. Epstein, B. L. Marks, R. L. Stiller, and R. G. Jacob, "The effect of nicotine on energy expenditure during light physical activity," The New England Journal of Medicine, vol. 320, no. 14, pp. 898-903, 1989.

[43] N. L. Benowitz and D. S. Sharp, "Inverse relation between serum cotinine concentration and blood pressure in cigarette smokers," Circulation, vol. 80, no. 5, pp. 1309-1312, 1989.

[44] H. C. Bucher, L. Bujanda, J. M. Marimon et al., "Mortality and light to moderate alcohol consumption after myocardial infarction," Lancet, vol. 353, no. 9160, pp. 1272-1273, 1999.

[45] J. A. Iestra, D. Kromhout, Y. T. van der Schouw, D. E. Grobbee, H. C. Boshuizen, and W. A. van Staveren, "Effect size estimates of lifestyle and dietary changes on all-cause mortality in coronary artery disease patients: a systematic review," Circulation, vol. 112, no. 6, pp. 924-934, 2005. 


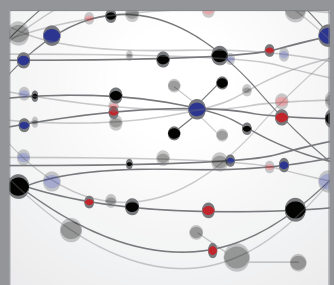

The Scientific World Journal
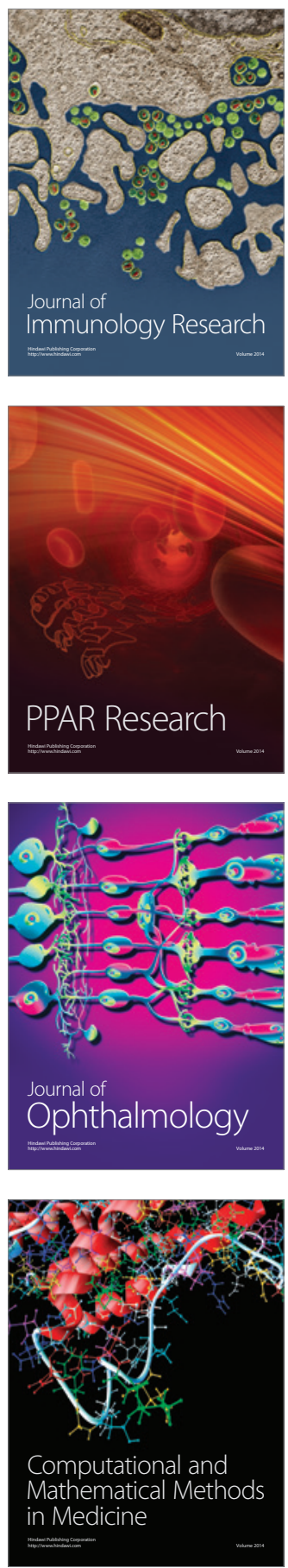

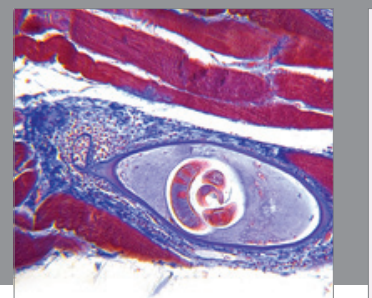

Gastroenterology

Research and Practice
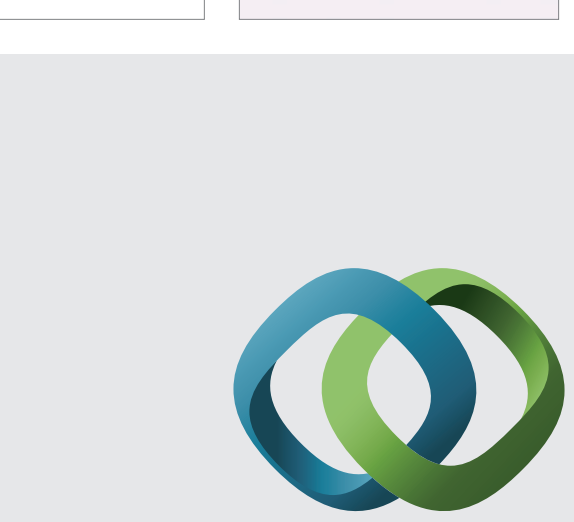

\section{Hindawi}

Submit your manuscripts at

http://www.hindawi.com
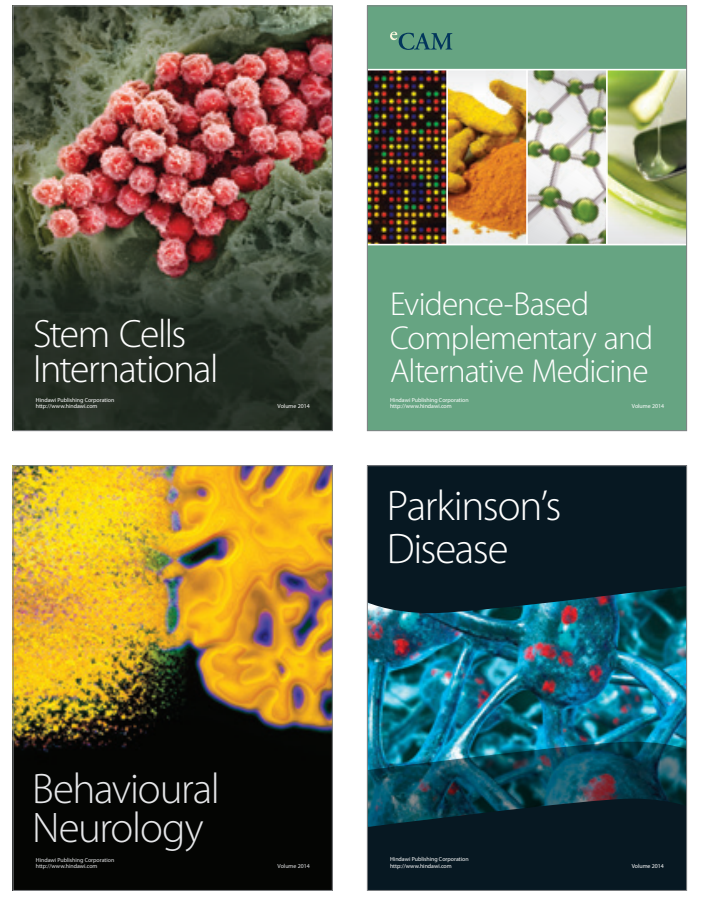
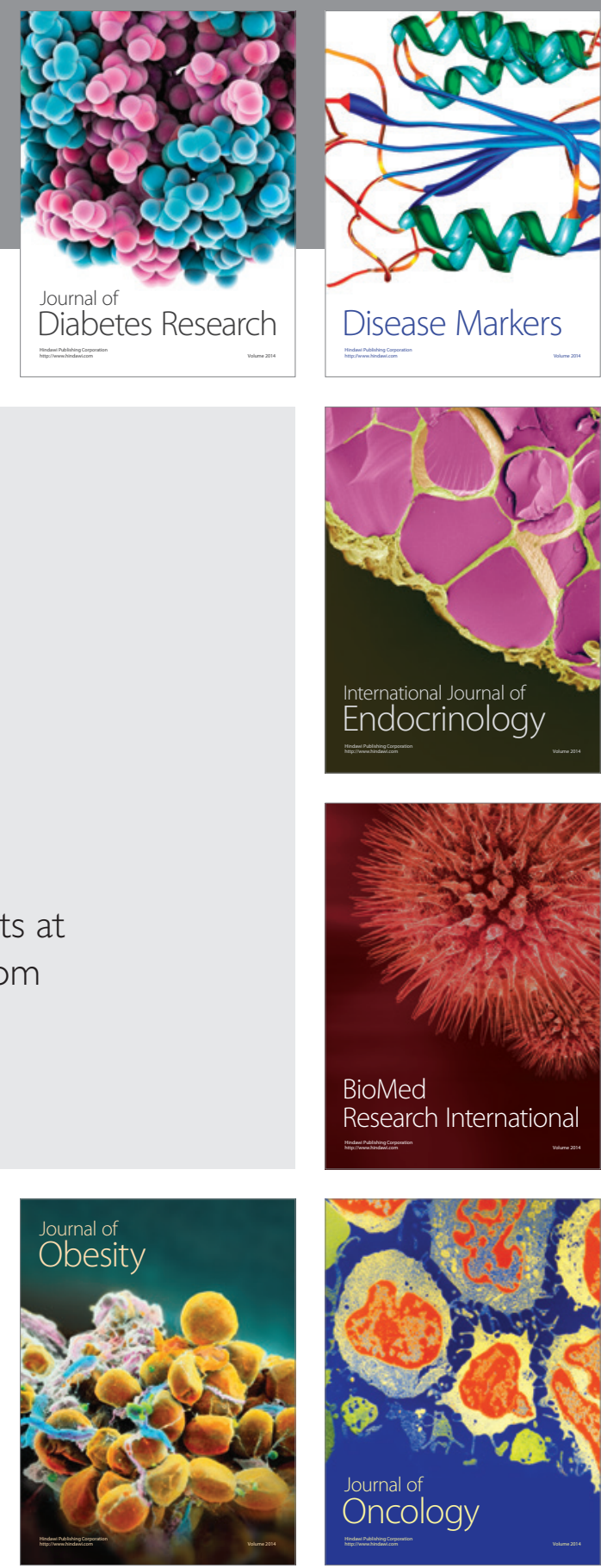

Disease Markers
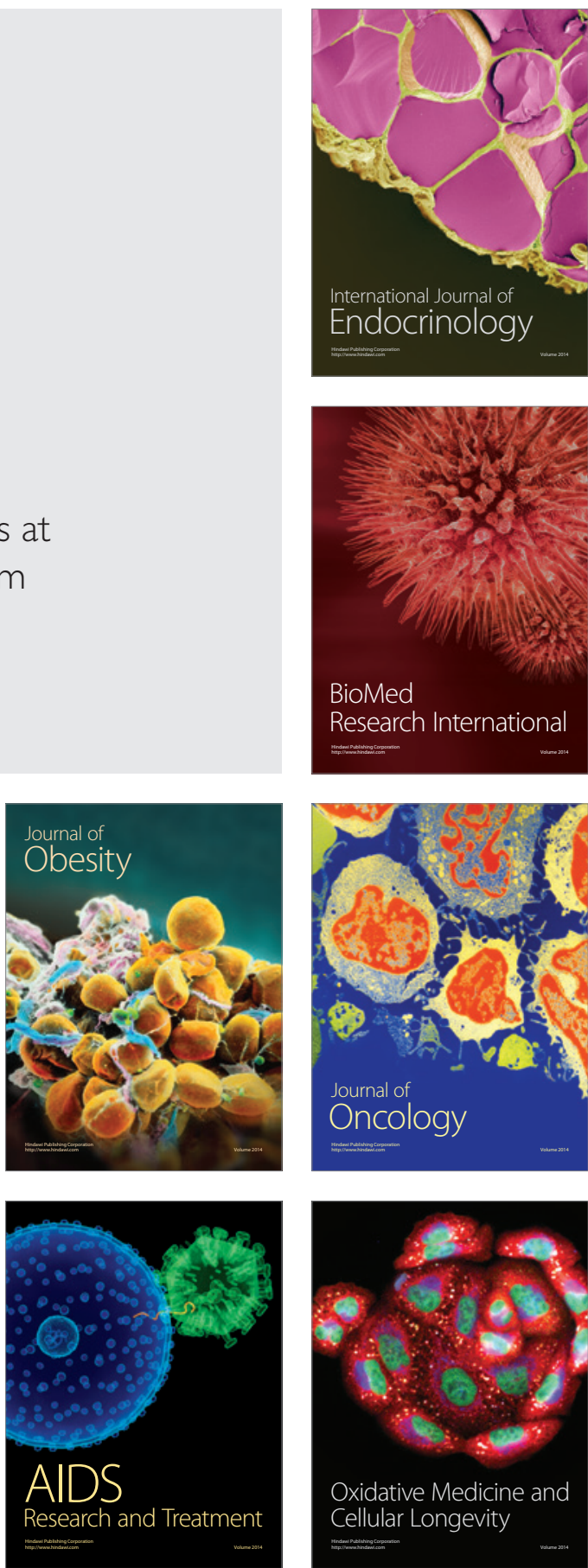